\title{
19
}

\section{WEB-Based Enhanced Services}

\author{
C. Rheinart
}

Compagnie IBM France

Le Plan du Bois

06610 La Gaude

France

Tel. $(+33) 492114929$

Fax. (+33) 493247821

E-mail: rheinart@vnet.ibm.com

\begin{abstract}
This article describes a prototype of integration of the Telephony and Internet domains for the enabling of new and existing Services for access via WWW protocols by operators, subscribers, or both. The described solution enables telephone subscribers who also have a WWW browser to perform:

- Self-registration for the use of a Telco Multiple Services Platform, and

- Self-registration and service management of single number call forwarding service.
\end{abstract}




\section{INTRODUCTION}

Web-Based Enhanced Services is a generic term for the enabling of new and existing services for access via WWW protocols by operators, subscribers, or both.

This solution is to be installed by a telephone company and enables telephone subscribers who also have a WWW browser to perform:

- Self-registration for the use of a Telco Multiple Services Platform (MSP) supporting various enhanced services (Voice, Fax, Conferencing,...), and

- Self-registration and service management of an innovative single number call forwarding service (Follow-me), via Java-based and e-commerce technology.

This solution allows the telephone company to offer these two new services with minimal requirement for operator services support. The benefits of this approach are:

- Reduces need for operator provisioning/support of enhanced telephony services, network management services.

- Reduces the subscriber's dependence on operators for provisioning and managing Enhanced Services.

- Remaining operator consoles can also be replaced by more extensive Web interfaces, thus allowing operator support to be provided from any Web browser on the telephone company's intranet, and reducing the need for specialised operator consoles.

- Eliminates touch-tone user interface as an obstacle to Enhanced Services.

- Enables wide range of new services at the intersection of the Internet and telephony.

This Web-based approach may be extended to a wide range of enhances services, which correspond to the implementation of the Computer-Telephone Integration (CTI) on a public network. A new generation of compact, portable devices which operate both as a mobile telephone and as an intelligent data terminal is emerging. The subscriber would then have a portable device which can both manage the Enhanced Services as well as make use of them.

In the following, we will consider some of the issues encountered by both the Telco Operator and the subscriber regarding the introduction of new Enhanced services. Then, we will describe the technical approach which has been selected for the development of the Follow-me application as regards to the self-registration and subscriber data provisioning functions. At least we will mention the difficulties encountered with this approach. 


\section{POSITIONING}

A Multiple Services Platform is an example of a network computing systems which allows a Public Network Operator to flexibly introduce advanced services. Enhanced services, for example, include:

- Voice applications (voice mail, calling cards, voice activated dialling, call forwarding, etc...)

- Fax applications (fax mail, never-busy fax, etc...)

- Data applications providing services accessible via a Personal computer

Moreover, a Multiple Services Platform may act as a Intelligent Peripheral (IP), controlled by an external intelligence (Service Control Point, SCP) within the Intelligent Network Architecture. IBM MSP/6000 is an example of such a multiple services platform.

Enhanced Services are often, but not always, provided by a telephone company, and are then usually closely integrated with the Central Office (C.O.) switches. Figure 1 shows a simple conceptual model of a Multiple Services Platform (MSP), which conforms to European approaches to Enhanced Services. Here the called party number, that is the dialled number, causes the voice channel to be routed by the C.O. switch to a front end switch controlled by the MSP Call Manager. Moreover, the C.O. switch notifies the Call Manager of the call details via a Common Channel Signalling System 7 or other interface. The Call Manager determines which MSP application should handle the call. For example, the MSP allocates voice mailboxes to specific processors, and to deposit a voicemail for a specific subscriber, the voice channel is routed to the processor for that subscriber's mailbox (or to an alternate).

In the same way, if a calling party calls the "single number" for an Enhanced Services subscriber with a single number call forwarding service, the Call Manager recognises the called number as a forwarded number and hands the call off to the forwarding service application, which runs on one of the Enhanced Services (ES) processors. The forwarding service determines where to forward the call, places a call to that number, and connects the incoming call when the subscriber responds.

Enhanced Services platforms thus provide valuable services for subscribers and are quite profitable for the telephone carriers. One of their chief disadvantages is that they are expensive and time-consuming to operate because they demand significant support from telephone operators in provisioning, configuring, managing and updating the service for each subscriber. As a result they are difficult to scale up. In principle, some of this support could be performed by the subscribers; for example, adding or deleting barred numbers in a calling barring service. However, the only interface available to the subscriber is the telephone instrument itself; so these support operations turn into either obscure code sequences, such as *70 (which typically disables call waiting), which subscribers cannot remember, or into frustrating exercises in DTMF menu navigation. More complex services such as configuring single number call forwarding are too 
complex to manage reliably by this means. The result is that the telephone company must provide operator support for the provisioning, configuration, management and updating of these services.

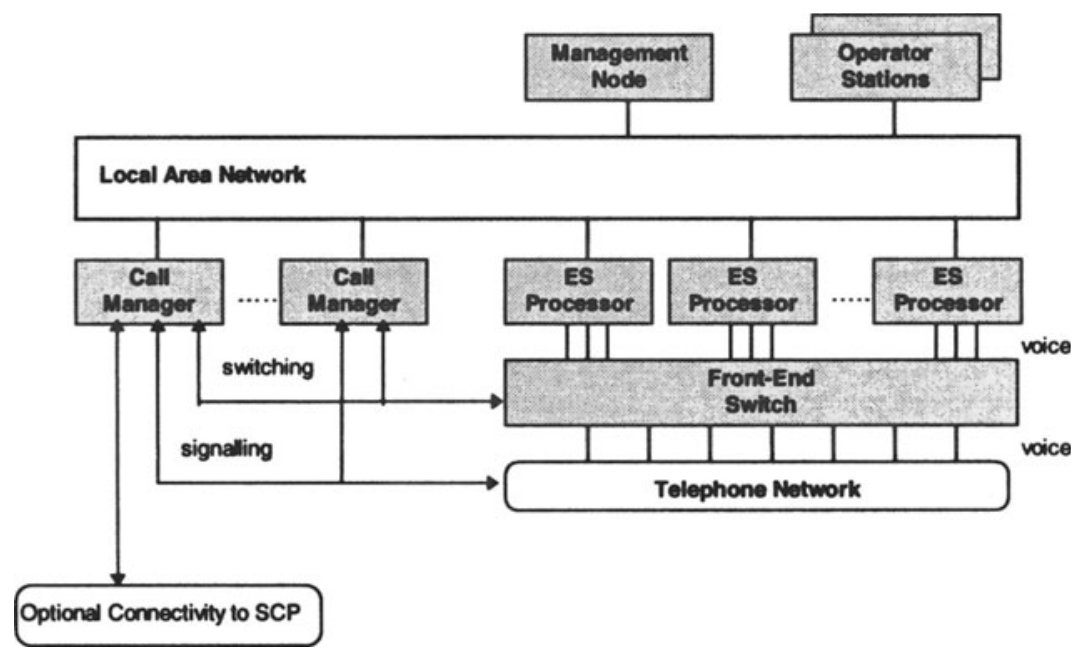

Figure 1 Conceptual model of a Multiple Services Platform (MSP).

Increasingly however, both consumer and business subscribers have access to the Internet and can make use of a Web browser. By some estimates, there are of the order of 100 million Web browsers in existence world-wide (after 3 years). Compare this with of the order of 1 billion telephone lines world-wide (after 125 years). A natural step is therefore to enable at least these subscribers to perform support operations on their Enhanced Services via the Web. Recently mobile telephones incorporating palmtop computers with built-in Web browsers have become available from AT\&T and from Nokia, making these natural devices for subscriber management of Enhanced Services.

Figure 2 shows a conceptual extension of the MSP for this purpose. If the Enhanced Services platform is made accessible via the telephone company's own intranet, the subscriber can gain network access to the Enhanced Services platform (modulo security provisions) by one of three paths:

- Dial-up access via the company's own gateway. This points to the close relationship between Enhanced Services and Internet service.

- Access via some other Internet Service Provider and then via the Internet itself.

- Broadband access via a leased, packet-switched connection to an enterprise, or, in the future, via an ADSL or HFC access network.

Here the Enhanced Services platform is supplemented with a WWW server platform. The Web server is provided with an application interface to the subscriber-manageable parts of the Enhanced Services; that is, most of the functions previously performed by the telephone operators, typically using 
specialised operator consoles. Of course, the operator consoles can also be replaced by more extensive Web-interfaces, thus allowing operator support to be provided from any Web browser on the telephone company's intranet, and reducing the need for specialised operator consoles

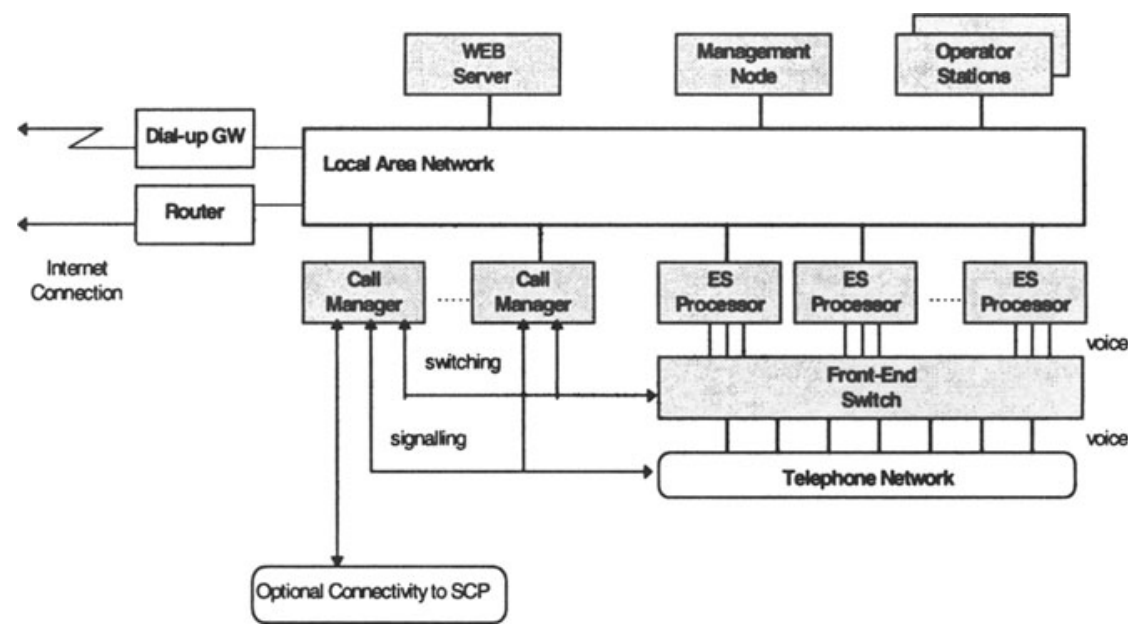

Figure 2 Conceptual model of Web-based Enhanced Services

While this is an interesting first step, this Web-based approach could eventually be extended to a very wide range of Enhanced Services. In essence, this approach is the implementation of Computer-Telephone Integration (CTI) on a public network, as opposed to a PBX, and one could eventually implement many of the advanced services that a PBX-based CTI system provides. For example, one might use a Web browser to access a White Pages telephone directory; clicking on a name in the directory would set up a PSTN call from the subscriber to the selected party. Similarly, an enterprise home page could display an 800 number, which is dialled when clicked. Contemporary CTI client applications are often based on the Microsoft Telephony API (TAPI), but these involve dedicated Windows applications and user interfaces. Today is emerging a range of Java object classes, which will fulfil the same function for a Web browser Java application that TAPI provides for a Windows application. 


\section{TECHNICAL APPROACH}

Figure 3 shows a software structure for the prototyping of a WES application.

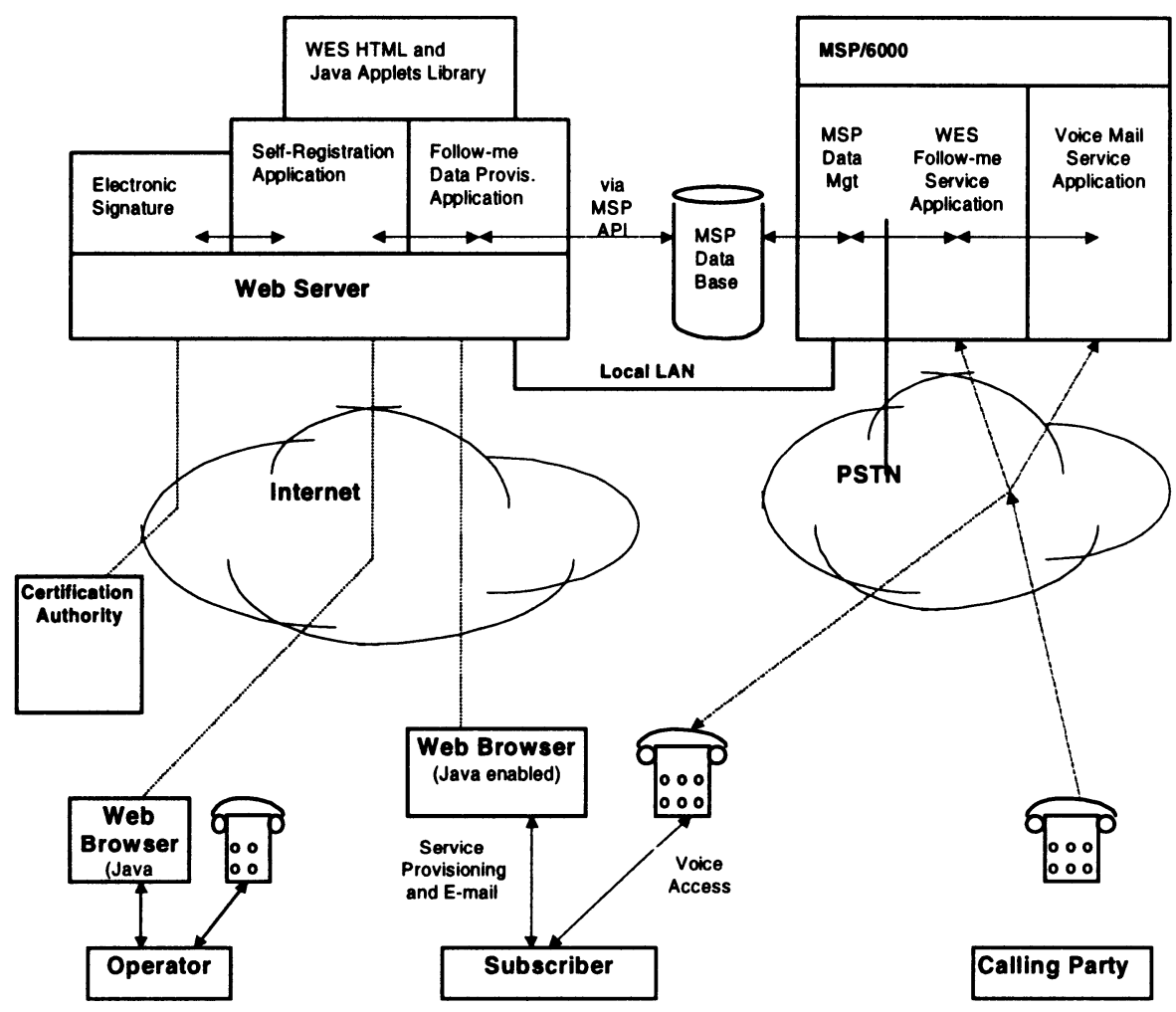

Figure 3. Software structure for the prototyping of a WES application

The client side consists of a standard Java-enabled Web-browser, which is supplemented by Java applets downloaded from the WES server. The client processor is not defined, except that it must have some access to the Internet. The server side runs on an RS/6000, which may be an existing part of the Enhanced Services platform. The WES server communicates with one or more subscriber applications, namely in our case, with the Self-Registration and Follow-me Data provisioning applications. The subscriber applications communicate either with MSP platform which implements the actual Enhanced Services on the MSP, or with external services such as a credit agency for electronic commerce. Associated with the subscriber applications are HTML JavaScript content for the Web pages, and Java applets to provide additional function at the client side.

The user navigates to the home page of the telephone company's Enhanced Services and logs in. The user is identified by his Subscriber Identification associated to his Personal Identification Number (PIN) which is a kind of password. The browser and server establish a session based on Secure Sockets and 
the server downloads the appropriate set of applets. The user selects the specific Enhanced Service he or she wishes to use and the applets establish the session with the appropriate WES server application. The subscriber may now perform SelfRegistration or modify his or her personal single profile, and so forth.

\section{SINGLE NUMBER CALL FORWARDING SERVICE}

In this service, the user establishes a daily or hourly calendar which defines where his or her telephone calls should be finally delivered. The user gives out a single number to his customers or co-workers or family, but calls to that number are routed according to a schedule established by the user. The user can also specify multiple numbers to be tried in sequence. For example:

- 07:00-08:00 Mobile, Voicemail

- 08:00-09:00 Office, Voicemail

- 10:00-11:00 Voicemail

- 11:00-12:30 Branch office, secretary, voicemail

- 12:30-01:15 Mobile, Voicemail

- 01:15-06:00 Office, Voicemail

- 06:00-07:00 Mobile, Voicemail

- 07:00-08:00 Voicemail

Here the user has defined one or more numbers where he or she may be reached at different times of a particular day.

When the user is called, the Follow-me determines how the call is to be handled. If the call has to be forwarded, the Follow-Me service dials each of the numbers specified for that time of day until the user answers; if the user does not answer, the calls will eventually be forwarded to voicemail. When one of the called numbers is answered, Follow-Me might request the responding person to accept or not the call by prompting for the input by DTMF (Touch-tone) key of a digit. Acceptance or not of the call might be coupled to a call screening function, which would consist in replaying an announcement message recorded from the calling party. The responding party would accept or not the call according the tonality of the voice.

Meanwhile the calling party receives a pre-recorded message that Follow-Me is searching for the called party, and the calling party can at any time opt to be transferred to voicemail. In a first step the out-dialled calls are billed to the called party, but call details are provided in the Call Data Record to fit specific carrier needs. This is a valuable service for mobile workers, even if they have mobile telephones, and is worth of Web-based implementation, because it is too complex to set up via a DTMF UIF.

As far is concerned the definition of the single number, several possibilities exist. This number can be: 
- One number among a range of real telephone numbers that would be allocated by the carrier to the MSP. During the self-registration process, MSP would allocate one of these numbers to the subscriber.

- One number among a range of logical telephone numbers that would be rerouted by the carrier to a fixed forwarding number (corresponding to MSP). This rerouting of the call could be done with the assistance of an Intelligent Network Service Control Point. During the self-registration process, MSP would allocate one of these logical numbers to the subscriber.

- The main telephone number of the subscriber, which appears on his business card for instance, and which would be rerouted by the carrier to a fixed forwarding number (corresponding to MSP) in case of no answer. Again the rerouting of the call could be done with the assistance of a Service Control Point. During the self-registration process, the subscriber would specify this single number.

Whatever the chosen alternative, calls to this single number are always delivered by the C. O. switch to the MSP. When the MSP Call Manager receives an incoming call with the associated signalling information (calling party number, called party number, original called party number and call reason), it identifies the call as a Follow-Me call, since it has a registry of the single numbers, and hands the call off to the Follow-Me service application.

The basic definition of the forwarding schedule is via a Web browser form. However, it may also be possible to enter or deduce the forwarding schedule from the subscriber's electronic calendar, for example, Lotus Organizer or the Lotus Notes calendar, if this is maintained on the personal computer.

\section{SUBSCRIBER SELF REGISTRATION}

We have noted above that a disadvantage of Enhanced Services from the telephone company's point of view is the high cost of operator support. A method of permitting the subscriber to establish the basic subscription to the MSP platform electronically is highly desirable and also original. Provisioning here means establishing the basic information about the subscriber: name, billing method, address, telephone numbers, and so forth. Generally this requires the subscriber to complete some paperwork, mail it to the company and wait a few days for the service to be enabled. Alternatively the subscriber may be able to call an operator number, give a credit card number as surety for the first bill, and establish a billing arrangement with the company. The first method involves a workflow process in the back office. The second method involves both an operator interaction and a workflow process in the back office.

A better method is to allow the subscriber to establish an e-commerce relationship with the company. This requires no human intervention. It also allows "on-demand" subscription to the services, permitting the subscribers to "try it and see". It also allows travellers to establish a local telephone number instantly and to 
subscribe to Enhanced Services such as voicemail and call forwarding for a few days at a time. Self-Registration thus simultaneously reduces the cost of provisioning and enable greater flexibility for the subscribers.

The service is implemented here as follows. The user indicates via a Web interaction the desire to become an MSP subscriber. The MSP Self-Registration service collects basic information about the subscriber, including a credit card number or other credit identification, which is transmitted securely to the SelfRegistration service via the Secure Sockets Layer (SSL) protocol.

Authentication of the credit information is verified via an electronic signature, in case the user has obtained such a signature from a Certification Authority like Verisign. Validation of the credit information can be done by a connection to the pertinent credit organism.

The Self-Registration service then creates the subscriber profile on the MSP and asks the subscriber what service(s) he or she wants to use. Thereafter, whenever an MSP service is activated on behalf of that subscriber, the MSP generates a Call Detail Record which is billed out (in real-time or not) to the subscriber's credit account via a financial switch. Subsequently, the subscriber can re-visit the SelfRegistration Web page and add or delete services or terminate the MSP subscription completely.

\section{WES OPERATOR}

We have seen that this solution allows the telephone company to reduce the need for operator provisioning/support of enhanced telephony services. However in a emergency or if the subscriber is unable to access the WES service via a workstation, the subscriber may call the MSP operator to perform certain actions:

- To become a MSP subscriber,

- To display/modify or cancel a current forwarding request,

- To enter a future forwarding request,

- To change his basic administration information such as his credit card number, his bank account, his e-mail address or even his home address,

- To request his Personal Identification Number, in case he has lost it,

- To cancel the Follow Me service subscription and so forth...

In this solution, the operator console for the enhanced telephony services is also a Web browser. This console is connected to the same Local Area Network as the Web Server. For security reason, communication with the Web server may use an access IP port different from the one used by the subscribers. When an operator on a WEB browser console wants to get access to the system, first of all, he has to authenticate himself as an operator. To each operator is allocated a specific operator-ID and a PIN number. You should consider an operator as a standard subscriber with specific privileges.

When receiving a call from a subscriber, the operator needs to handle two types of situations: 
- The subscriber is able to authenticate himself as a real subscriber, that is, he is able to provide his subscriber identification and his PIN number. This is the case when the subscriber does not have access temporary to a Web browser, or simply when he is lost while handling a forwarding request. The operator must be able to act as this subscriber. The implementation solution consists to give the operator the possibility to copy in his own context, the context of the calling subscriber. Some carriers may feel that the updating of some basic information related to the subscriber (credit card number, bank account, e-mail address or even the home address) should be performed via the operator to avoid frequent updates from the subscriber.

- The subscriber is NOT able to authenticate itself as a real subscriber: he has lost his subscriber identification or his PIN number. The operator is able to search in the database and to retrieve the requested information. The problem is how to transmit this information? The operator can not be sure of the person who is calling. The most secure way is to send it via mail (e-mail in our case), as it is done for any credit card. We know that e-mail is not a secure transport protocol, but the user can changed his PIN number as soon he has received the e-mail message.

As an extension of this solution, we can imagine a customer who does not have access to a WEB browser but who wants to take profit of the follow-me service. He may request the operator to perform the operations on his behalf. Nothing prevents the operator to do this. You should note in this case that the subscriber will receive his Subscriber-ID and PIN number by physical mail rather than by e-mail.

Indeed, the operator will also have to deal with requests coming from the carrier control operations. He needs to be able to perform the following actions:

- To display all information related to a specific subscriber,

- To cancel the registration of a specific subscriber for a specific service,

- To cancel the registration of a user as a MSP subscriber, and so forth...

\section{DIFFICULTIES}

\section{User Interface}

One of the main motivations of this prototype lied in the fact that the poverty of the user interface via the DTMF keys inhibited any service somewhat sophisticated, if it required a certain number of subscriber data. With a Web browser, a graphical interface allows indeed to simplify the user task. However a telephone user remains still today, in his culture, reluctant to spend a minimum amount time to manage his telephone calls.

With the same goal to minimise the time spent by the subscriber for data provisioning, Java applets allow indeed to save time for the local interactions, but the time spent to download the Java classes may be an inhibitor for the user having a low speed Internet connection, even when these classes are put together and compressed. 


\section{Self-Registration}

We have noted above that the self-registration process comprises two parts:

- self-register to the MSP platform itself,

- self-register to a new service.

\section{Self-registration to the MSP platform}

Via the subscriber-Id and the PIN number, the user is correctly identified and the subscriber data are conveyed in a secure way via the Secure Sockets Layer (SSL) protocol. The issue of the authentication of the subscriber remains. Nothing prevents a malicious user from specifying a credit card number and an address that would not belong to him, and it may apply even when the credit card is valid and has not been stolen. The issue gets even more complex because there is in our case no purchase of any material good which would allow an easier checking later on. However, the system may work like this, as it is the case today with most of the shopping over the Net, the minimum being to validate the credit card.

To go further on and to overcome the fear of many people, especially in some countries, to place their credit card on the Net, the solution is to use an electronic signature which can be obtained from a Certification Authority. The difficulty lies in the broad acceptance and usage of these facilities.

\section{Self-registration to a new service}

The introduction of a new service which needs data provisioning from the subscriber requires also often a provisioning action in the switches of the network. Generally speaking, to make automatic this C.O. update function requires a gateway connection with the Telco System Management Service. In the case of our prototype, this constraint is relaxed by selecting the Single Number values among a set of logical telephone numbers provided by the Telco, as described in chapter 'Single Number Call Forwarding service'. This approach did allow to provision the switches of the network in advance.

\section{Billing}

Several questions arise concerning the billing:

- How to charge the service?

- fee per service

- fee per service usage

- monthly fee.

- How often would the Telco want to charge the customer?

- Would the customer prefer ONE bill for all the services, including services he is using to day?

The answers to all these questions are Telco specific. However, it appears that a connection with the current Telco billing system is recommended, to get the required flexibility. 


\section{CONCLUSION}

The technology exists today to allow an Internet user to self-register to Telco enhanced services and to manage his personal data, while reducing to a minimum the role of the Telco service operator.

However, some cultural reluctance from the telephone user exists today:

1. to place his credit card number on the Net,

2. and to spend a certain amount of time to update his personal data.

Concerning the first point, until the electronic signatures facilities are widely deployed and well advertised to the users, the Telco service operator will keep a significant role for the delivery of the subscriber number with an associated password.

Concerning the second point, a Telco company could begin to offer on the Net a list of services which could be quickly activated and exercised by the user, while requiring little amount of user interactions. This would allow the user to get familiar with Internet habits and be prepared to accept more sophisticated applications.

\section{BIOGRAPHY}

CHARLES RHEINART graduated as engineer of the « Ecole Nationale Supérieure d'Ingénieur de Toulouse » in 1968 in the domain of Applied Mathematics. He has been working for IBM in the areas of networking, transmission protocols and network management for over 20 years. He participated in 1994 in a RACE project targeted to group collaborative working over broadband networks. He has joined in 1996 the IBM European Telecommunications Solution Center (TSC) architecture group located in La Gaude (France). His current area of interest is the integration of the Telephony and Internet domains. 